\title{
Geburt und Kindheit Christi.
}

Von H. Usener in Bon $\mathrm{n}$.

I

Die Lehre und das Leiden unseres Heilands waren längst Gegenstand schriftlicher Überlieferung geworden, ehe man dazu schritt, das Bild seines Lebens nach dem Anfang hin abzurunden. Nicht nur Marcus sondern sogar noch der, inhaltlich betrachtet, jüngste Evangelist Johannes setzen mit der Wirksamkeit des Täufers Johannes ein. Nur die Evangelien des Matthäus und Lukas berichten von der Geburt und Kindheit Christi. Aber diese beiden Berichte weichen weit, ja in unvereinbarer Weise von einander $a b$.

I. Matthäus erzählt I, 18-25 summarisch: Maria von Joseph gefreit ( $\mu \nu \eta \subset \tau \in U \theta \epsilon i c \eta c)$ sei, bevor die beiden Gatten sich vereinigt hätten (cuv$\in \lambda \theta \in \hat{v}$ : Heimführung oder eheliche Beiwohnung?), schwanger erfunden worden vom heiligen Geist; ihr Mann, da er auf das Gesetz hielt (díkaıc w้v) und doch sie nicht an den Pranger stellen wollte, habe den Entschluß gefaßt, sich in aller Stille von ihr zu trennen; da habe sich ihm

I Der folgende anspruchslose Versuch, von dem Stand der Fragen über die Geburt und Kindheit des Heilands eine knappe Übersicht zu geben, war für die Encyciopaedia biblica von T. K. Cheyne und J. S. Black geschrieben, wo er III S. $3340 \mathrm{ff}$. in englischer Bearbeitung erschienen ist. Auf den besonderen Wunsch des Herausgebers dieser Zeitschrift wird er mit freundlicher Erlaubnis des englischen Verlegers hier in seiner ursprünglichen Fassung vorgèlegt. - Von den Vorarbeiten seien hier die wichtigsten ein für allemal genannt: D. F. Strauß, Leben Jesu 1835 u. ö., für das deutsche Volk bearbeitet 1864. E. F. Gelpke, Die Jugendgeschichte des Herrn. Bern I841. P. Lobstein, Die Lehre von der übernatürlichen Geburt Christi, christologische Studie. II. Aufl. Freiburg i. Br. 1896. Alfred Resch, Das Kindheitsevangelium nach Lucas und Matthaeus unter Herbeiziehung der aussercanonischen Paralleltexte quellenkritisch untersucht. (- Texte und Untersuchungen zur Geschichte der altchristlichen Litteratur herausgeg. von O. v. Gebhardt und A. Harnack, Band X Heft IV). Leipzig 1897. Ludwig Conrady, Die Quelle der kanonischen Kindheitsgeschichte Jesus'. Göttingen Igoo. Joh. Hillmann, Die Kindheitsgeschichte Jesu nach Lucas, kritisch untersucht: Jahrbücher für protestantische Theologie XVII (1891) S. 192-261. A. W. Zumpt, Das Geburtsjahr Christi. Leipzig 1869.

Zeitschrift f. d. neutest. Wiss. Jahrg. IV. 1903. 
ein Engel des Herrn im Traume gezeigt mit den Worten 'Joseph Sohn Davids, scheue dich nicht, dein Weib Mariam zu dir zu nehmen (mapa$\lambda \alpha \beta \in \hat{i} v)$; denn was in ihr gezeugt ist, stammt aus dem heiligen Geiste; sic wird einen Sohn gebären und du sollst ihn mit dem Namen Jesus benennen, denn er wird sein Volk erretten von seinen Sünden'. Darin findet der Evangelist die Erfüllung der von Jesaias 7, 14 gegebenen Prophezeiung, welche nur in der Fassung der LXX ('Jungfrau' statt 'junges Weib') herangezogen werden konnte. Er fährt dann fort: Joseph habe, aus dem Schlafe erwacht, nach der Anordnung des Engels gehandelt, und sein Weib zu sich genommen, aber ihr nicht ehelich beigewohnt bis daß sie einen Sohn ( $C D L$ 'ihren erstgeborenen Sohn' nach Lc) geboren, den er Jesus nannte. Nun erst erfahren wir den Schauplatz und die Zeit dieser Vorgänge: es war Bethlehem in Judaea, wo Jesus geboren ward, und die Regierungszeit des Herodes (2, I). Die Göttlichkeit des Knäbleins wird alsbald durch ein Zeichen bekräftigt. Magier (ihre Zahl wird nicht genannt) aus dem Ostẹn trafen in Jerusalem ein und fragten: Wo ist, der (eben) geboren ist zum König der Juden: wir haben seinen Stern im Osten gesehn und sind gekommen, ihn zu verehren. Bestürzt über diese Kunde beruft Herodes alle Oberpriester und Schriftgelehrten, und diese erklären unter Berufung auf Micha 5, I Bethlehem in Judaea als den Ort, wo der den Juden verheißene Messias geboren sein müsse. Nachdem er von den Magiern sich über die Zeit, wo der Stern aufgegangen, unterrichtet, entläbt er sie mit dem Auftrage, genaue Erkundigungen über das Knäblein einzuziehen und ihm zu überbringen. Der Führung des Sternes folgend, bis er stehen geblieben, gelangen sie zu dem Hause Josephs (2, I I Gíc tìv oỉíav), finden das Knäblein mit seiner Mutter Maria, fallen vor ihm nieder, um es zu verehren, und überreichen ihm aus ihren Schatzkästen Gaben, Gold, Weihrauch und Myrrhen. Durch einen Traum gewarnt kehren sie jedoch nicht zu Herodes zurück, sondern wählen einen anderen Weg zur Heimreise. Auch dem Joseph erschien ein Engel des Herrn und mahnte ihn, mit dem Kind und der Mutter nach Ägypten zu fliehen, um den Nachstellungen des Herodes zu entgehen. Das tat er und blieb in Ägypten, bis Herodes gestorben war: wodurch sich das Wort des Osea II, I erfültte 'aus Ägypten habe ich meinen Sohn gerufen'. Herodes aber ließ in ohnmächtigem Grimme alle Kinder von zwei Jahren und darunter, der Zeitangabe der Magier entsprechend, in Bethlehem und Umgegend schonungslos töten. Das Zeichen zur Rückkehr aus Ägypten erhält Joseph wiederum durch einen Engel im Traume. Aber da er vernimmt, 
daß Herodes' Sohn Archelaos jetzt über Judaea herrsche, fürchtet er sich nach Judaea (d. h. nach Bethlehem) zurückzukehren, und wendet sich gemäß einer neuen Traumoffenbarung in das Galiläische Land, wo er sich in Nazareth ansiedelt.

Wenn man in Abzug bringt, was Schriftgelehrsamkeit aus dem alten Testament herangeholt hat, bleibt in diesem Bericht des Mt nichts übrig, was nicht aus lebendiger Überlieferung, will sagen aus volkstümlicher Sage geschöpft sein könnte. Ja die Ungenauigkeiten und Unklarheiten, welche den Erklärer stören und am schärfsten von Conrady hervorgehoben sind, scheinen eine schriftliche Vorlage auszuschließen und erklären sich am ungezwungensten aus der sorglosen Wiederholung mündlicher Überlieferung.

2. Einen ganz verschiedenen Eindruck macht der Bericht des Lucas I, 5-2, 50. Er ist ein Erzeugnis schriftstellerischer Kunst, und diese Kunst macht sich geltend nicht nur in der Nachbildung hebräischer Psalmenform, sondern vor allem im ganzen Aufbau der Erzählung. Der Verfasser baut seine Geschichte auf den durch das Evangelium gegebenen Voraussetzungen auf, daß die Tätigkeit Johannes des Täufers vorbildlich dem Auftreten Jesus' voranging und daß in Jesus der von den Juden erwartete Messias erschienen war; die Geschicke der beiden, des Heilands und seines Vorläufers, sucht er nun von ihrer Geburt, ja vom Mutterleibe an mit einander $\mathrm{zu}$ verschlingen.

Ausführlich wird erzählt, wie dem greisen Priester Zacharias, während er den Tempeldienst versieht, der Engel Gabriel erscheint und ihm verkündet, daß seine bis in ihr hohes Alter zur Unfruchtbarkeit verurteilte Frau Elisabeth ihm einen Sohn gebären werde, der im Geiste und in der Kraft des Elias vor dem Herrn einherziehen und ihm sein Volk bereiten werde. Die ungläubige Verwunderung des Zacharias wird durch plötzliche Unfähigkeit der Sprache bestraft. Sein Weib aber wird schwanger und hält sich fünf Monate vor den Leuten verborgen. $\mathrm{Da}$, während diese in ihrem sechsten Monate ging, erschien derselbe Engel Gabriel der nit Joseph verlobten aber noch unvermählten Maria, um ihr die Botschaft zu überbringen, daß sie empfangen und einen Sohn gebären werde, dem es bestimmt sei auf dem Throne seines Vaters David in Ewigkeit zu herrschen. Verwundert, da sie sich noch Jungfrau weil, wird sie vom Engel belehrt, daß sie ihre Leibesfrucht dem heiligen Geiste verdanken werde. Zugleich wird sie auf das bevorstehende Mutterglück ihrer Verwandten Elisabeth hingewiesen. In ihrem Glücksgefühl sucht Maria die Verwandte im Gebirgsland Judaea's auf und wird 
von dicser prophetisch als die Gebenedeite des Herrn begrüft; vor Freuden hüpft selbst der ungeborene Johannes im Mutterleibe. Es folgt dann der dem Lobgesange der Hanna (I Sam 2, If.) nachgebildete, etwas sehr unbestimmte, aber ccht hebräische Psalm, das berühmte Magnificat ( $\mathrm{I}, 46-55)$. Nach den Bemerkungen Hillmanns (Jahrb. f. prot. Theol. XVII $197 \mathrm{ff}$ ) hat D. Völter (Theologisch tijdschrift XXX 254-G) den zwingenden Beweis erbracht, daf dieser Psalm nicht, wie die handschriftliche und kirchliche Überlieferung will, der Maria, sondern der Elisabeth gehört, und Harnack hat kürzlich die Frage zum Abschluß gebracht, indem er sowohl das handschriftliche Maria als das an dessen Stelle von manchen alten Zeugen gelesene Elisabeth als glossematischen Eindringling und als echte Überleitung zum Psalm lediglich die Worte kai єi் feststellte, als deren Subjekt sich nun durch den Zusammenhang von selbst Elisabeth ergibt (Sitzungsberichte der Berliner Akademie 1900 N. XXVII S. 538 ff.). Nach drei Monaten kehrt Maria zurück und es ereignen sich nun die Vorgänge bei der Beschneidung und Namengebung des Johannes, die unerwartete Wiederkehr der Sprache bei Zacharias und sein Lobgesang ( $1,67-79$ ), der gleichzeitig dem Messias und dem Sohne, der diesem die Wege bereiten soll, gerecht wird. Mit einem kurzen Satze über die Jugend und den Aufenthalt des Johannes in der Wüste schließt der Vorbericht über Johannes.

Die Geburt Jesus' zu Bethlehem wird durch die von Augustus für das ganze Reich angeordnete, vom Statthalter Syriens Quirinius (Kupívıoc) in Palästina durchgeführte Schatzung begründet, welche Joseph mit seinem Weib, weil er zum Hause Davids gehört, in die Davidstadt Bethlehem zu reisen nötigt. Dort wird das junge Weib von ihrem ersten Sohne entbunden, den sie in der Krippe niederlegt. Die Hirten auf dem Felde, durch die Verkündigung des Engels und den Jubelruf der himmlischen Heerscharen getrieben, kommen und preisen das Knäblein in der Krippe, und Maria merkt sich ihre Worte in bedächtigem Herzen. Wie das Gesetz es vorschreibt, wird der Knabe nach acht Tagen beschnitten und erhält dabei den vom Engel vorgeschriebenen Namen

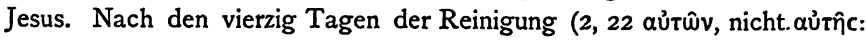
auch der Mann ist durch die Berührung mit der Wöchnerin befleckt) erfolgt das Opfer und die Darbringung des Erstgeborenen im Tempel zu Jerusalem, durch welche dem greisen Symeon, dem es verheißen war vor seinem Ende den Messias zu schauen, und der Prophetin Anna Gelegenheit gegeben wird die Verwirklichung ihrer Hoffnungen $z \mathrm{u}$ bezeugen. Nun erst, nachdem allen Vorschriften des Gesetzes Genüge 
getan ist, können die Eltern mit ihrem Kinde die Rückreise nach Nazareth antreten. In ungestörter Entwicklung verläuft dort mit der Gnade Gottes die Jugend des künftigen Heilands. Nur ein Ereignis der Jugendzeit hat der Evangelist wert erachtet zu schildern, die Scene, wie der Zwölfjährige im Tempel zu Jerusalem von den Eltern unter den Lehrern der Schrift betroffen wird.

Nach seiner ganzen Haltung, den leitenden Gesichtspunkten, der wiederholt angewandten Form des hebräischen Psalms, der guten Kenntnis jüdischer, der mangelhaften römischer Verhältnisse läßt, wie allgemein anerkannt ist, dieser Bericht die Hand eines Judenchristen nicht verkennen. Die Erzählung zerfällt auch innerlich in zwei deutlich von einander geschiedene Hälften, die Vorgeschichte des Johannes (c. I) und die Geburt und Kindheit Jesus' (c. 2). Während in der ersteren Zacharias und Elisabeth im Vordergrund stehen und die Empfängnis der Maria nur episodisch dazwischen tritt, ist in der zweiten von jenen und ihrem Sohne Johannes nicht mehr die Rede. Aber beide Teile von einander zu trennen, wie man wohl vorgeschlagen hat, ist unmöglich. Sie sind fest zusammengefügt; der Lobgesang des Zacharias weist auf den Erlöser hin, und in den prophetischen Worten des alten Symeon wiederholt sich dieselbe Form des hebräischen Psalms, die in den Hymnen der Elisabeth und ihres Mannes hervortritt. Freilich der Raum, welcher der Vorgeschichte des Johannes zugebilligt wird, steht sichtlich nicht in dem Verhältnis zur Hauptsache, der Verkündigung an Maria, das wir bei einem Schriftsteller erwarten dürfen, der selbständig die Feder ansetzt, um die Menschwerdung des Heilands zu schildern. Es ist sehr möglich, daß die wunderreiche Erzählung von der Verkündigung und Geburt des Johannes, also das, was Lc I, 5-25. 46-55. 57-80 steht, in dem Kreise der Johannesjünger gedichtet und verbreitet war, bevor es mit der Vorgeschichte Jesus' verknüpft wurde. Wäre die Dichtung, welche die Anfänge des Erlösers und seines Vorläufers in einander schlingen sollte, aus einem Gusse, so würde sie den Eltern des Heilands breiteren Raum und stärkere Betonung gewährt und nicht die Hauptgestalten in den Schatten der Nebenfiguren gestellt haben. Die Offenbarung an Zacharias (I, I4-I7) verkündet in dem wiederkehrenden Elias den Vorläufer nicht sowohl des Heilands als Gottes selbst (s. Malachias 4, 5), und der Lobgesang der Elisabeth ist ohne erkennbare Beziehung auf Jesus. Es würde unter jener Annahme die ganze Anlage des I. Kapitels sich bestens erklären; der judenchristliche Verfasser des Ganzen würde dann diese Dichtung eines Johannesschülers mit Überarbeitung des dem 
Zacharias in den Mund gelegten Psalns einfach übernommen, durch die Verkündigung und den Besuch der Maria erweitert und in dem kurzen Hymnus des Symeon $(2,29-32)$ gewissermaßen nachgeahmt haben. Soviel wird man Völter (Theol. tijdschrift 30, $244 \mathrm{ff}$.) unbedenklich zugcben dürfen, ohne darum seinen kritischen Einzelergebnissen beizupflichten.

3. Jedes ungetrübte Auge wird sehen, daß die Geburtsgeschichten des Mt und Lc einander ausschließen und unvereinbar sind.' Was ihnen gemeinsam ist, die Personen Josephs, Marias und Jesus', die Berufung Jesus' zum Messias, die Zeit des Herodes und die Geburt in Bethlehem, sind gegebene Voraussetzungen. Aber auf dieser Grundlage werden von beiden Evangelisten ganz verschiedene Gebäude errichtet. Die Heimat Josephs ist bei Mt Bethlehem, bei Lc Nazareth, die Göttlichkeit Christi wird bei Lc durch die Verkündigung des Engels an die Hirten und den Lobgesang der himmlischen Chöre, bei Mt durch den Aufgang des Sterns im Osten bezeugt; dem neugeborenen Messias wird bei Lc von den Hirten des Feldes, bei Mt von den Magiern die erste Verehrung dargebracht. Die Familie des Heilands flüchtet bei Mit vor dem Wüten des Herodes nach Ägypten und schützt sich dann vor Archelaos durch die Übersiedelung nach Nazareth, während sie bei Lc nach Vollzug der durch die Erstgeburt auferlegten Pflichten sofort nach Nazareth zurückkehrt. Und dort verläuft die Jugend des Heilands ungestört, während bei Mt die ersten Lebensjahre durch die Gefahr und den Wechsel des Wohnorts beunruhigt werden. Ein noch tieferer Gegensatz gegen Mt wird sich ergeben, wenn wir den Bericht des Lc von einem jüngeren Zusatz befreit haben werden.

Selbstverständlich hat der Glaube die beiden kanonischen Berichte hinnehmen und mit einander verarbeiten müssen. Schon bei Justinus Martyr beginnt diese Arbeit theologischer Vermittelung. Den Widerspruch zwischen dem göttlichen Ursprung Jesus' und der evangelischen Tatsache, daß nicht Maria, sondern Joseph dem Hause Davids zugeschrieben wird, gleicht er dadurch aus, daß er Maria aus dem Hause Davids stammen läßt (Dial. 43. 45. 100) und das Davidische Geschlecht Josephs mit der Angabe verschleiert, daß er zum Stamme Juda gehört habe (Dial. 78). Er läßt (ebend.) den Joseph von Nazareth, wo er wohnte, nach Bethlehem, wohẹ er war, reisen, um die widersprechenden Heimatsangaben $z \mathbf{u}$ beseitigen. Es ist von Interesse, gleichzeitig mit diesen ersten' Vermittelungsversuchen auch schon Fortbildung der

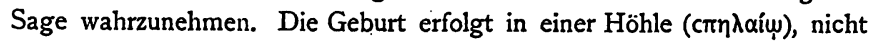


im Stalle (Dial. 78) , und die Magier kommen bei ihm bereits aus Arabien (so öfter). Reicht das zu, um mit Credner (Beiträge zur Einleitung in die biblischen Schriften I, 212 ff.) und anderen, die ihm nachsprechen, die Geburtsgeschichte des Justinus auf eine außerkanonische Quelle zurückzuführen?

Noch vor dem Ende des zweiten Jahrhunderts ist, um den verdrießlichen Widerstreit der beiden Evangelien aufzuheben, in freier, durch Kenntnis jüdischer Verhältnisse nicht eingeschränkter Fabulistik ein Urevangelium geschaffen worden, das sogen. Protevangelium Jacobi, ein apokryphes Schriftchen, das große Verbreitung gefunden und namentlich der Malerei früherer Jahrhunderte Anregung gewährt hat. Es war sicher dem Origenes, möglicherweise schon dem Clemens von Alexandreia bekannt. Obwohl der Verfasser weit über unsere Evangelien zurückgreift und die Vorgeschichte der Maria mit göttlichen Wundern zu umgeben sucht, verrät er doch im übrigen keine andere Absicht als die beiden Berichte unserer Evangelien, so weit es angeht, in wörtlicher Abhängigkeit zu vereinigen und auszugestalten. Die Abhängigkeit tritt gerade da am stärksten hervor, wo der Verfasser sich von dem Evangelium zu entfernen scheint. Ganz der Geschichte der Maria zugewandt hat er die bei Lc damit verschlungene Vorgeschichte des Johannes auszumerzen versucht; aber die Begegnung der Maria mit Elisabeth mochte er nicht missen (c. 12), und das Verstummen des Zacharias erwähnt er als etwas Bekanntes, ohne die Ursache angegeben zu haben oder das Ende dieses Zustandes zu berühren. Verräterisch ist auch die ungeschickte Einfügung der Magierepisode. Die wenigen Abweichungen von dem Evangelium fallen dagegen nicht ins Gewicht. Die Geburtshöhle ist wie bei Justinus aus volkstümlicher Überlieferung übernommen; die Unterdrückung der Flucht nach Ägypten, die nur durch Interpolation 22, 2 in einzelnen Manuskripten steht (s. auch c. 25), mag auf Nachgiebigkeit gegen das vom Verfasser bevorzugte Evangelium des Lc beruhen. Harnack hat (Geschichte der altchristl. Literatur I 19, II 598 f.)

Diese volkstümliche Überlieferung ist auch in das Evangelium eingedrungen, aber seltsamerweise nicht sowohl bei Lukas als bei Matthaeus 2, 9. Wie mich Herr Preuschen freundlich belehrt, hat Conybeare in Hastings Dictionary of the Bible I 154a darauf hingewiesen, daß die genannte Stelle nach der ältesten armenischen Evangelienhand-

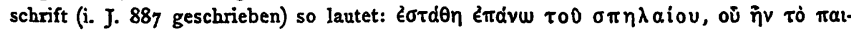

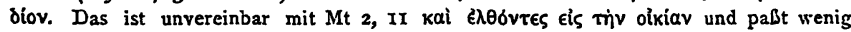
zu dem ganzen Bericht des Mt, der von außerordentlichen Verhältnissen des Elternpaars zur Zeit der Geburt nichts weib. 
diesem Produkt apokrypher Fabulistik im wesentlichen gerechte Beurtcilung zuteil werden lassen.

Andere apokryphe Quellen der Geburts- und Kindheitsgeschichte findet man in der Ausgabe der Evangclia apocrypha von Const. Tischendorf (ed. II Lips. 1876); über ihren Inhalt berichtet R. Hofmann, Das Leben Jesu nach den Apokryphen (Leipz. 1851). Aber eine selbständige Darstellung ist nach dem Protevangelium nicht mehr hervorgetreten. Alle ferneren Behandlungen der Geburtsgeschichte berulien lediglich auf den drei genannten Quellen, den Evangelien und jenem Apokryphon, wie L. Conrady gezeigt hat (Quelle der Kindheitsgeschichte S. $172 \mathrm{ff}$.). Was hinzugekommen ist, hat, teilweise durch die Liturgie angeregt, wie den Ochs und Esel an der Krippe, die Volksdichtung geschaffen.

Für die offenbarungsgläubige Theologie mußte die Vereinbarkeit der widerstrebenden Berichte stets ein Dogma sein. Sie erklärt die Divergenz der Quellen daraus, daß jeder der beiden Evangelisten sich verschiedene Abschnitte derselben Geschichte zur Erzählung ausgewählt habe. In dem erleuchteten Zeitalter der Quellenkritik·mußte sich natürlich diese Vorstellungsweise trotz der Mahnung Schleiermachers (Leben Jesu IVW. I $6 \mathrm{~S}$. $50 \mathrm{ff}$.) zu der Hypothese auswachsen, dab hinter Mt und Lc eine einheitliche Quellenschrift stehe, ein Evangelium von der Geburt und Kindheit Jesus'. Es ist das zweifelhafte Verdienst von Alfred Resch, dies "Kindheitsevangelium" gefunden und gleich auch griechisch und hebräisch wiederhergestellt zu haben. Mein Freund L. Conrady hat auf dieser abschüssigen Bahn noch einen Schritt weiter getan und versucht, das Protevangelium des Jakobus als die gesuchte einheitliche Quelle zu erweisen.

\section{II}

I. Zu einer Kritik dieser Darstellungen geben die Evangelien selbst genügenden Anhalt. Trotz den Überarbeitungen, die sie vor ihrer kanonischen Fixierung erfahren mußten, sind in unseren Evangelien nicht selten Beziehungen auf Verhältnisse, die mit jüngerem Zuwachs unver-. einbar sind, treu bewahrt worden, in der Regel wohl darum, weil sie unlöslich verknüpft waren mit bedeutsamen Aussprüchen unseres Herrn, die man nicht missen mochte und nicht streichen durfte. Es ist längst。 und oft bemerkt worden, daß wie Paulus so auch die Evangelien selbst nicht das geringste von der wunderbaren und göttlichen Geburt des Heilands wissen. Wohl aber ist ihnen das natürliche Sohnesverhältnis des Heilands zu dem Zimmermann Joseph und seinem Weibe Maria 
noch genau bekannt. Selbst die Episode von dem Aufenthalt des 12 jährigen Jesus im Tempel zu Jerusalem (Lc 2, 4I-50) kennt nur dies Sohnesverhältnis: 'und seine Eltern wußten es nicht' (43), was zeitig in 'Joseph und seine Mutter' umgeschrieben wurde, und Maria sagt zu Jesus v. 48 'dein Vater und ich haben dich mit Schmerzen gesucht'. Zwar hat die Episode den Zweck, zum ersten Male das Bewußtsein der Sohnschaft Gottes hervorbrechen zu lassen (v. 49); aber gerade daß

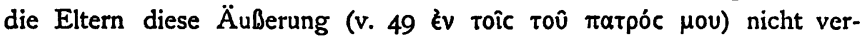
stehen, ist ein zwingender Beweis dafür, daß für die Vorstellung des Erzählers Joseph und Maria die leiblichen Eltern Christi waren und sich als solche wußten. Noch deutlicher redet der alte Bestand der Evangelien. Als der Heiland nach seiner grundlegenden Tätigkeit am Galiläischen See einmal in die Heimatsstadt Nazareth kam und dort in der Synagoge auftrat, fragten die Leute verwundert: 'Ist das nicht des Zimmermanns Sohn? heißt seine Mutter nicht Mariam ('Ist das nicht der Zimmermann, der Sohn der Maria?' Mc bereits überarbeitet) und seine Brüder Jakobus und Joseph und Simon und Judas? und sind nicht seine Schwestern alle bei uns?' (Mt I3, 55 f. vgl. Mc 6, 3). An der entsprechenden Stelle des Lc 4, $22 \mathrm{f}$. nimmt Jesus die Frage der Nazarener 'Ist das nicht Josephs Sohn?' ruhig als berechtigt hin und lehnt nur Wundertätigkeit mit der Bemerkung $a b$, daß der Prophet in seinem Vaterland nichts gelte; die Prophetenstelle (Jes 6I, I), die er dort verliest, spricht von der Salbung durch den h. Geist, aber nicht von Sohnschaft Gottes. Im Ev. Johannis werden die Zweifel an dem messianischen Berufe Jesus' dem Nathanael aus Bethsaida in den Mund gelegt; sein Bruder Philippos hatte ihm gemeldet, der Messias, von dem Moses und die Propheten geschrieben, sei gefunden, Jesus der Sohn des Joseph aus Nazareth' $(I ; 45)$, und er antwortete 'kann denn aus Nazareth etwas Gutes kommen?' Am beredtesten spricht die synoptische Erzählung von der Verleugnung der Seinigen (Mt 12, 46-50 Mc 3, 31-35 Lc 8, 19-21), diè allein Mt im ursprünglichen Zusammenhang aufbewahrt hat. Die Wunder, die Jesus tat, hatten die von den Schriftgelehrten geförderte Vorstellung erregt, daß Jesus mit dem Teufel einen Bund geschlossen habe, 'durch den Obersten derr Teufel treibt er die Teufel aus' (Mc 3, 22 vgl. Mt 12, 24 Lc I1, I5 Joh I0, 20). Auch in das Elternhaus war diese Nachricht gedrungen, und bestürzt machten sich seine Brüder mit der Mutter auf den Weg, um sich seiner zu bemächtigen und ihn mit sich nach Haus zu nehmen, sie dachten nicht anders als er sei irre geworden (Mc 3, 2I). Sie fanden ihn in einem Hause 
inmitten einer Menge Volks, das ihm lauschte, und vermochten nicht zu ihm durchzudringen. Als sie ihn bitten ließen zu ihnen heraus zu kommen, sprach cr, auf die Schüler und Hörer weisend, das Wort 'Siehe, das ist meine Mutter und meine Brüder' u. s. w., ein Wort, dessen Schroffheit durch dic Überzeugung verständlich wird, daß er bei den Seinigen Verständnis für seine Tätigkeit nicht finde: im Ev. Joh 7,5 wird das mit dürren Worten von den Brüdern Jesu bezeugt. Auch die Apostelgeschichte crwähnt mit der Mutter die Brüder Jesus' (1, 14). Zu dem Ergebnis dieser Beobachtungen stehen in bestem Einklang die beiden Geschlechtsregister, welche uns Mt I, I-16 und Lc 3, 23-38 erhalten haben. Beide sind vollständig unabhängige Versuche, Jesus in genealogischen Zusammenhang mit David zu bringen, aber beide wurzeln in der Voraussetzung, daß Jesus der leibliche Sohn Josephs sei, und haben nur unter dieser Annahme $Z_{\text {weck }}$ und Sinn. Während Mt die Liste in drei Perioden mit der typischen Zahl von je 14 Geschlechtern von Abraham bis auf Joseph und Jesus herunterfuhrt, steigt Lc von Jesus, 'der ein Sohn, wie man glaubte, Josephs war' aufwärts bis zu Adam, 'der ein Sohn Gottes war'; nur die ersten 14 Stammväter des Mt und zwei im Eingang seiner dritten Periode (Salathiel und Zorobabel) kehren bei Lc wieder. Der Zusatz bei Lc 'wie man glaubte' und der Schluß der Liste des Mt 'Joseph der Mann der Maria, von der ( $\xi \xi \bar{\eta} c)$ Jesus, der den Namen Christus führt, geboren ist' verraten die Hand ausgleichender Überarbeiter; aber den unvereinbaren Widerspruch der auf Joseph, nicht auf Maria gestellten Geschlechtsreihen mit der Geburtsgeschichte haben diese zaghaften Korrekturen nịcht zu heben vermocht. Wenn wir Lc Glauben schenken, so steht Maria überhaupt dem Hause Davids fern: sie heiBt 'Venvandte' (currevíc Lc I, 36) der Elisabeth, diese war jedoch 'von den Töchtern Aarons' (I, 5). Daß aber Clemens Romanus im I Brief an die Korinthier 32, 2 Jesus als einen geborenen Leviten (durch Maria natürlich) betrachte, darin vermag ich Hilgenfeld und Hillmann (Jahrb. f. prot. Theol. I7, 250 f.) nicht zu folgen. Erst als die jungfräuliche Geburt Christi durchgedrungen war, konnte man sich genötigt fühlen, Maria aus dem Hause Davids stammen zu lassen; das tut schon Justinus martyr (S. 6), dann das Protevangelium des Jakobus c. ro, sogar in den syrischen Palimpsest vom Sinai ist Lc 2, 5 (4) die Interpolation 'weil sie beide aus dem Hause Davids waren' eingedrungen. Das ist alles ebenso selbstverständlich, wie daß die theologische Interpretationskunst eines Resch das davidische Geschlecht Marias aus den Evangelien selbst herausliest (Kindheitsev. S. I9I). 
2. Nur hinweisen will ich auf die chronologischen Schwierigkeiten, an deren Hebung der gelehrte Scharfsinn von Jahrhunderten sich vergeblich abgemüht hat. Wenn Mt den Heiland unter der Herrschaft des Königs Herodes geboren werden und erst, nachdem diesem sein Sohn Archelaos in der Herrschaft Judaeas gefolgt ist, aus Ägypten zurückkommen läßt, so wird dadurch die Geburt Christi um einige Jahre über das Jahr 4 vor Chr., das Todesjahr des Herodes zurückgeschoben. Lc dagegen knüpft die Geburt Christi an die nach Befehl des Augustus in Palästina unter P. Sulpicius Quirinius, dem Statthalter Syriens vollzogene Schatzung. Diese war nicht denkbar vor der Absetzung Archelaos' 6 nach Chr. und wird tatsächlich für diese Zeit bezeugt durch Josephus Ant. Jud. XVII I3, 5 (355) und XVIII I. Wenn es nun auch zu großer Wahrscheinlichkeit erhoben worden ist, daß Quirinius schon vorher in der Zeit von 3-2 vor Chr. die Provinz Syrien verwaltet hatte (s. Mommsen Mon. Ancyr. ${ }^{2}$ p. I6I ff. E. Schürer, Gesch. des jüdischen Volkes I² 260 ff.), so kann doch der für den Bericht des Lc wesentliche Umstand, die durch Quirinius vorgenommene Schatzung, nicht von dem Jahre $6 \mathrm{n}$. Chr. verrückt werden. Lc steht aber nicht nur mit Mt, sondern auch mit sich selbst in Widerspruch, da auch er im Anfang seines Berichtes ( $I, 5$ ) das Ereignis in 'die Tage Herodes des Königs der Juden' verlegt. Beide Ansätze sind durch einen $Z$ wischenraum von mehr als Io Jahren von einander getrennt. Eine brauchbare Zeitbestimmung läßt sich aus den Berichten über die Geburt Jesus' überhaupt nicht gewinnen. Ungefähr läßt sich das Jahr der Geburt nur durch die Angaben des Lc 3, I. 23 ermitteln, daß Johannes im 15. Jahre des Tiberius 28/29 n. Chr. hervorgetreten und Jesus im Beginne seiner Wirksamkeit, also 29, ungefähr dreißig Jahre alt gewesen sei. Man findet die Zeugnisse für die verschiedenen darauf gebauten Berechnungen übersichtlich bei Clinton Fasti Hellenici 3, 260 ff. zusammengestellt; die letzte Untersuchung der Frage hat A. W. Zumpt (1869) geliefert.

Über den Tàg der Geburt schweigen die Evangelien. Die Kirche hat ihn mittels mythologischer Analogetik festgestellt. Während die alte Kirche, und so noch immer die armenische, die Geburt am Feste der Epiphanie (6. Januar) feierte, das aus dem alexandrinischen Fest der Erscheinung des Dionysos abgeleitet ist, hat die römische Kirche nach der Mitte des IV. Jahrh. den natalis Solis invicti d. h. den 25. December als Geburtsfest des Heilands durchgesetzt. Aber vorher waren die verschiedensten Ansätze versucht worden. Der gelehrte Jesuit Antonmaria Lupi hat sich das Vergnügen gemacht (Dissertazioni, lettere ed altre 
operette, Faenza 1785, 1, 219 ff.) zu zeigen, daß es keinen Monat im Jahre gibt, in den nicht die Geburt gesetzt worden wäre.

3. Ein anderer Widerspruch, den wir nicht unerwähnt lassen dürfen, betrifft den Ort der Geburt. Es war eine ebenso feste UUberlieferung, dab Jesus zu Bethlehem geboren war, wie dab er aus Nazareth stammte. Bcide Evangelisten haben sich bereits mit diesem Widerspruch abfinden müssen. Mt nahm Bethlehem einfach als die Heimat Josephs und lief diesen erst nach der Rückkehr von Ägypten aus Furcht vor Archelaos seinen Wohnort zu Nazareth in Galiläa nehmen; er hatte kein Recht 13, 54 Nazareth die 'Vaterstadt' ( $\pi \alpha \tau$ í $\delta \alpha$ ) Christi $2 \mathrm{u}$ nennen, wie der Vorgänger (Mc 6, I) es durfte. Lc betrachtet Nazareth als Heimat Josephs, und bedient sich, um die Geburt zu Bethlehem zu motivieren, des Auskunftsmittels der Schatzung. Wie die Lehrtätigkeit des Heilands bis zur letzten Reise nach Jerusalem sich wesentlich im Umkreise des benachbarten Galiläischen Sees bewegte, so stand auch seine Herkunft aus Nazareth in Galiläa fest (Mc 6, I-4 Mt 13, 54 f. Lc 4, I6ff. Mt 21, I I. 26, 69. 71 Joh I, 46. 7, 4I); ja Mt 2, 23 hat dafür sogar ein freilich sehr apokryphes Prophetenwort in Bereitschaft: 'auf dafi erfüllet werde, was durch die Propheten gesagt ist: Er wird ein Nazoräer heißen'. Wie konnte Bethlehem damit in Konkurrenz treten?

Man hat daran erinnert, daß auch in Galiläa ein Bethlehem unweit von Nazareth lag, im Talmud einmal Bethlehem Noseriyyal genannt. Unsere Frage kann durch die Einführung dieses zweiten Bethlehem nicht entwirrt sondern nur weiter verwickelt werden. Denn es ist ebenso gewi $\beta$, da $\$$ das Bethlehem, in welches unsere Evangelien die Geburt Christi verlegen, das judäische südlich von Jerusalem.ist, wie als Heimatsort desselben Nazareth feststand. Aber wichtig ist, daß dieses Bethlehem nur den Berichten über. Christi Geburt bekannt ist. Hier liegt der Schlüssel. Nach der Predigt vom Wasser des Lebens werden, wie es im Ev. Joh 7, 40 f. heißt, verschiedene Stimmen des Volkes laut. 'Da sagten etliche ... Dies ist in Wahrheit der Prophet. Andere sagten: Dies ist der Christus. Andere sagten: Kommt denn der Christus aus Galiläa? hat nicht die Schrift gesagt, daß der Christus aus dem Samen Davids und von Bethlehem, wo David war, komme?' Schon beim Einzug in Jerusalem ruft das Volk Jesus als 'dem Sohne Davids' sein Hosianna zu (Mt 2I, 9 vgl. 15 Mc II, ro) und die Pharisäer wissen, daß der Gesalbte des Herrn nur ein Sohn Davids sein könne

I Cheyne in dem Artikel 'Nazareth' der Encyclop. biblica 3, 3360 ff. 
(Mt 22, 42 Mc 12, 35 Lc 20, 41). Aus der Prophezeiung des Micha 5, I war die weitere Forderung abgeleitet, daß der Messias aus der Davidstadt Bethlehem hervorgehen müsse. Die Schriftgelehrten, welche Herodes bei Mt zu Rate zieht, können angesichts dieser Prophezeiung (Mit 2,6) gar nicht in Zweifel sein, wo der neugeborene König der Juden zu suchen ist. Die Erzählung des Johannes, eines Evangelisten, der die wunderbare Geburt noch nicht kennt, stellt die tatsächliche Heimat des Heilands, Galiläa, in Gegensatz zu der durch den jüdischen Glauben geforderten Geburtsstätte des Messias, und enthüllt uns den verborgenen Weg, auf dem Bethlehem in die Überlieferung der Evangelien gekommen ist. Schon zu Lebzeiten wurde Jesus als der 'Gesalbte des Herrn' betrachtet, Petrus selbst hatte die Bezeichnung geprägt (Lc 9, 20 vgl. Mc 8, 29; bei Mt 16, I6 'du bist der Christus, der Sohn des lebendigen Gottes'). (Die ganze Vorstellungsreihe, die sich den Juden an den Begriff des Messias knüpfte, mußte notwendig auf Jesus übergehn, sobald erst die Auffassung, daß er der 'Christus' sei, durchgedrungen war: das ist das Naturgesetz der Legendenbildung. Vor allem mußte Jesus ein Nachkomme Davids, also aus königlichem Stamme sein. Schon vor seinem Tod hat man diese Folgerung gezogen. Die erste literarische Wirkung waren die Geschlechtslisten, welche Jesus' Vater Joseph mit David in Verbindung setzten. So nüchterne Prosa sie reden, sind sie doch die ältesten Versuche der Dichtung über die Geburt Christi. Der nächste unvermeidliche Schritt war, seine:Wiege nach Bethlehem zu versetzen. Als die Berichte des Mt und Lc abgefaßt wurden, war das bereits ein fester Glaubenssatz geworden, der wohl oder übel mit der geschichtlichen Heimat Jesus vermittelt und ausgeglichen werden mußte.

Die Widersprüche mit der durch das Evangelium selbst verbürgten Wahrheit beweisen, daß zu der Zeit, wo die Geburts- und Kindheitsgeschichte hinzugefügt wurden, der Kern der Evangelien des Mt und Lc bereits feststand. Diese Zutaten müssen ganz anderen Federn entstammen als das übrige - den Inhalt meine ich natürlich, nicht die Form. Denn die Möglichkeit bleibt durch unsere sachliche Kritik unberührt, daß wir die heutige Form einem Überarbeiter verdanken, der die verschiedenen Bestandteile bereits vorfand. Daran scheint Harnack nicht gedacht $\mathrm{zu}$ haben, als er unlängst in den Sitzungsberichten der Berliner Akademie 1900 N. XXVII S. $547 \mathrm{ff}$. aus der Gleichheit des Sprachgebrauchs und des Wortschatzes glaubte beweisen zu können, dalb die beiden ersten Kapitel des Lukas von einer und derselben Hand herrührten wie das ganze übrige Evangelium und die Apostelgeschichte. 
Nicht einmal jene zwei ersten Kapitel können als zweifellos einheitliche Schöpfung eines einzigen Kopfes betrachtet werden. Das entscheidende Wort muß der sachlichen Kritik und der Analyse der Komposition vorbehalten bleiben. Während bei Mt der sachliche Widerspruch des Evangeliums dic Kindheitsgeschichte als Eindringling erkennen läbt, sind wir bei Lc in der Lage, das aus der Kritik des Inhalts abgeleitete Urteil durch das Zeugnis des Verfassers selbst erhärten zu können. Seine Berufung auf dic, 'welche von Anfang an Augenzeugen und Diener des Wortes gewesen' (Ev. I, 2 vgl. 3 àvw $\theta \in v$ ) würde auch ohne die ausdrückliche Erklärung dieses $\alpha \pi^{\prime}$ ' $\rho x \eta \hat{c}$ und ăvw $\theta \in v$, die er Apostelg. 1, 22

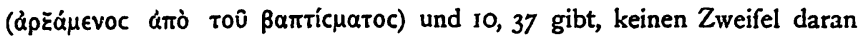
lassen, dab Lc sein Evangelium erst mit der Taufe und Predigt des Johannes begonnen hat. Das hat P. Corssen im wesentlichen richtig und überzeugend dargetan (Göttinger gel. Anz. 1899 N. 4 S. 315-327).

\section{III}

Die ältesten Niederschriften des Evangeliums wußten nichts anderes, als daß Jesus zu Nazareth geboren war als Sohn des Joseph und der Maria, aber sie lehrten auch, daß Jesus der von den Propheten verkündete, von den Juden erwartete Messias sei, und sie hatten davon zu berichten, wie Christus selbst von dem Bewußtsein erfüllt war, Gottes Sohn zu sein. Mit diesen Vorstellungen waren die Keime gegeben, die in den empfänglichen Gemütern der alten Christengemeinde fruchtbaren Boden fanden und verhältnismäßig rasch sich zu dem Dogma von der Göttlichkeit der Person Christi, ja von der Präexistenz des Sohnes Gottes entwickeln mußten.

I. Von dem Messias erwartete der jüdische Glaube nicht übernatürliche Geburt; er mußte nur aus dem Hause Davids stammen und von Gott erwählt sein (vgl. Hillmann Jahrb. f. prot. Theol. I7 [189r], 233 ff.). Daraus hatte sich unweigerlich als erste Folgerung, wie wir gesehen, ergeben, daß der Vater Jesus ein Abkomme Davids und daß Jesus in Bethlehem geboren sein mußte. Es folgte aber notwendig auch, daß der Auserwählte Gottes mit Gott selbst in nähere Beziehung gesetzt wurde. Der als Mensch geboren und herangewachsen war, bedurfte einer göttlichen Weihung $z u$ seinem Berufe. So entstand die Dichtung von der Jordantaufe.

In den Eingang der ältesten Evangelien war das Auftreten Johannes des Täufers, seine Predigt und Taufe gestellt. Durch das Vorbild des Täufers wurde Jesus zu seiner großen Aufgabe erweckt; den unverkenn- 
baren Nachhall des tiefen Eindrucks, den der Täufer auf ihn gemacht, zeigt die begeisterte Anerkennung, die Jesus ihm spendet (Mt II, $7 \mathrm{ff}$. Lc 7, 24-35 vgl. Mt 21, 32). Erst auf die Kunde, daß der Tätigkeit des Johannes seine Gefangennehmung durch Herodes ein vorzeitiges Ende bereitet hatte, tritt Jesus aus dem bisherigen Dunkel hervor (Mt 4, I2 Mc I, I4). So hindert nichts anzunehmen, da sich am Jordan zu Johannes drängte, um getauft zu werden, sich auch Jesus befunden hatte, und $\mathrm{da} b$ diese Tatsache schon von Anfang in den Evangelien erzählt gewesen war. Diese Taufe aber gab die erwünschte Gelegenheit, aus dem Menschen Jesus den Gesalbten des Herrn zu machen. Es ist in doppelter Weise geschehen. Nach Mc I, Iof. sieht Jesus, als er aus dem Jordan emporsteigt, den Himmel sich spalten, den heiligen Geist in Gestalt einer Taube sich auf ihn herablassen, und eine Stimme hört er vom Himmel: 'Du bist mein geliebter Sohn, an dem ich Wohlgefallen habe'. Diese aus dem Urtext (nicht LXX) von Jes 42, I abgeleiteten und auch bei der Verklärung auf dem Berge angewendeten Worte sollen Gott selbst bezeugen lassen, daß er Jesum zum Messias auserwählt habe; und der Geist Gottes geht in ihn ein, um das Wort des Jesaias 42, I. II, 2 zur Wahrheit zu machen. Kühner verfuhr derjenige, welcher die kurze Nachricht von Jesus Jordantaufe dem Lc-Evangelium 3, 2I f. einfügte. Er begnügte sich nicht mit der göttlichen Berufung zum Messias, sondern wollte auch für die Sohnschaft Gottes ein unmittelbares göttliches Zeugnis. Er benutzte dazu die Worte des Psalmisten 2, 7 (vgl. Apostelg. 13, 33) und lieb Gott sagen: 'Mein Sohn bist du, heute habe ich dich geboren.' Die griechische Kirche hat so bis etwa 300 , der lateinische Westen bis über 360 die Lc-Stelle gelesen; die Vorstellung selbst hat dazu geführt, die Menschwerdung Gottes an die Jordantaufe zu knüpfen; das Fest der Epiphanie, der Erscheinung Gottes auf Erden hat überall, bevor das Weihnachtsfest durchdrang, gleichzeitig der Taufe und der Geburt Christi gegolten. Gleichsam im Wetteifer mit dieser volltönenderen Bildersprache wurde die andere Fassung des Wunders am Jordan mit weiteren Wundern ausgestattet, wie es das Altertum im Mt und dem Hebräerevangelium las; auch das Ev. des Johannes geht über den ursprünglichen Bericht weit hinaus.

2. Die mythischen Bilder, die damit geschaffen waren, konnten gläubige Herzen auf die Dauer nicht befriedigen. Der immer mehr durchdringenden Vorstellung von der Göttlichkeit Jesu widerstrebte es, die Weihung zum Messias oder die Adoption zum Sohne Gottes erst in das dreißigste Lebensjahr des Heilands zu verlegen. Er mußte von 
Geburt an das crwählte Werkzeug Gottes sein. So erwuchs die Sage von Christi Geburt. Sic ist crst zu einer Zeit entstanden und ausgebildet worden, als mit der Jordantaufe bereits die Weihung zum Messias sich fest verbunden hatte. Wären beide zu etwa gleicher Zeit oder die Geburtsgeschichte vorher entstanden, so hätte das Taufwunder nicht dic Gestalt erhalten können, die es heute hat, oder wäre nie ausgebildet worilen; das eine schliebt das andere aus.

Auch hier konnte cin doppelter Weg beschritten werden. Wie bei der Jordantaufe göttliche Bezeugung der göttlichen Zeugung gegenüber steht, so konnte auch hier neben der wunderbaren Erzeugung Christi eine mchr der jüdischen Denkweise entsprechende Darstellung geschaffen werden, in welcher dem menschlichen Sohne des Joseph und der Maria bei der Empfängnis und Geburt göttliche Offenbarungen die Erwählung zum Messias bezeugten.

Eine solche Darstellung liegt tatsächlich bei Lukas vor. Wenn wir die Beobachtungen festhalten, die wir bei Bethlehem machen konnten, werden leicht die Fesseln, die lange Gewöhnung an geheiligte Überlieferung um uns legt, gelöst werden. Es ist das Verdienst Joh. Hillmanns (Jahrb. f. prot. Theol. 17, 221 ff.) mit zwingenden Gründen dargetan zu haben, daß die beiden Verse des Lc 1, 34-35, die einzigen, worin dort die göttliche Geburt Jesus' aus der Jungfrau Maria angekündigt wird, mit der ganzen übrigen Darstellung der Kap. I-2 unvereinbar sind, also von einem Überarbeiter eingefügt worden sein müssen. Nach Ausscheidung dieser Stelle bleibt eine rein judenchristliche, noch ganz auf der alten und echten Überlieferung, daß Jesus der Ehe des Joseph und der Maria und zwar als Erstgeborener entsprossen sei, fußende Erzählung von der Geburt des Messias übrig, und es findet sich nun kein Wort darin, was nicht aus den jüdischen Vorstellungen von dem kommenden Messias seine volle Erklärung erhielte.

Der Engel Gabriel kommt, von Gott gesandt, nach Nazareth zu einer Jungfrau Maria, die mit Joseph, einem Nachkommen Davids ( $\xi \xi$ olkou $\Delta$ aueío I, 27) verlobt war; er überrascht sie durch seinen Gruß und verkündet ihr dann, daß sie schwanger werden und einen Sohn gebären werde, der Sohn des Höchsten genannt und auf dem Throne Davids, seines (Stamm-)Vaters sitzen werde u. s. w. ( $\mathrm{I}, 3 \mathrm{I}-33$ ), woran er dann die Mitteilung von der eingetretenen Schwangerschaft der bis ins Alter unfruchtbaren Elisabeth, ihrer Verwandten schliebt (I, $36 \mathrm{f}$.). Die Geschehnisse im Hause der Elisabeth (I, 39-56), auch der Psalm des Zacharias ( $\mathrm{I}, 68 \mathrm{ff}$.) dienen nur dazu, den Messias schon im Mutter- 
leibe $\mathbf{z u}$ verherrlichen und die Beziehungen des künftigen Johannes zu ihm vorzubereiten. Nahe vor dem Ende der Schwangerschaft Marias erfolgt dann die übel durch die Schatzung motivierte Reise des Ehepaares nach Bethlehem. Joseph muß sich in der Stadt Davids zur Eintragung in die Steuerrolle stellen, weil er 'aus dem Hause Davids' stammt $(2,4)$, und zivar samt 'seinen Weibe Maria': denn nichts anderes als cùv Mapıò̀ Tn̂ ruvaıki aủroû las v. 5 noch der von Missis Agnes Smith Lewis entdeckte syrische Palimpsest vom Sinai und cum MTaria uxore sua bezeugen die vorhieronymianischen Texte von Verona und Vercelli, auch der Colbertinus, eine Lesung, die ganz abgesehen von dem Gewicht der Zeugen für sich selbst spricht; dafür wurde dann inter-

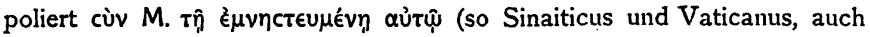
die lat. Übers. von Brescia), und wie so häufig sind zeitig beide Lesungen

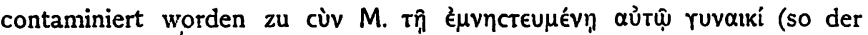
Alexandrinus, die lat. Übers. von Corbie, und schon Eusebios und Kyrillos Katech. I2, 3I); daß hier wirklich Contamination stattgefunden hat, sieht man recht anschaulich an dem alten Freisinger Ms. (Old-latin biblical texts 3,75 ) cum maria uxore su[a] desponsata ei, wo die alten Varianten

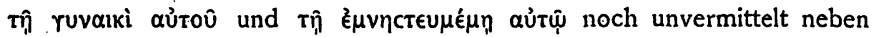
einander stehen. Da nun im Eingang der Erzählung I, 27 zweimal nach einhelliger Überlieferung hervorgehoben wird, daß Maria bei der Botschaft des Engels, obwohl dem Joseph verlobt, noch Jungfrau war, so können wir mit Sicherheit aus 2, 5 folgern, daß in der ursprünglichen Gestalt des Berichtes noch die kaum entbehrliche aber für den Überarbeiter, der I, $34 \mathrm{f}$. einfügte, unerträgliche Bemerkung nach I, 38 gestanden hat, daß danach Maria von Joseph heimgeführt worden sei und empfangen habe; dazu stimmt es bestens, wenn 2, 2 I daran erinnert wird, daß der Name Jesus von dem Engel vorgeschrieben worden sei, bevor er empfangen wurde im Mutterleibe'. Daß Jesus die erste Frucht dieser legitimen Ehe gewesen, wird mit klaren Worten 2, 7 gesagt 'und

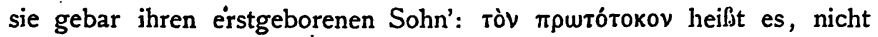

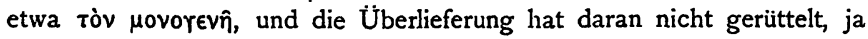
es ist sogar in Mt I, 25 interpoliert worden. Jesus wird also anerkannt als der älteste unter den Söhnen und Töchtern Josephs, welche das Evangelium selbst nicht vergessen hat. Nach jüdischem Ritus findet dann nach acht Tagen Beschneidung und Namengebung statt $(2,2 \mathrm{I})$, nach vierzig Tagen Weihung des Erstgeborenen und Opfer im Tempel zu Jerusalem (2, 22 f.): der ganze Vorgang setzt natürliche Geburt aus legitimer Ehe voraus, und ausdrücklich wird das 2, 27 bestätigt 'da die 
Eltern das Kind Jesus hereinbrachten'. Ganz im Sinne der Messias-Verheillung, die in den Worten des Engels sowohl zu Maria ( $1,31-33)$ als zu den Hirten (2, II vgl. 14) erklungen war, sind die Begrübungen des alten Symeon $(2,29-35)$ und der Prophetin Anna $(2,36-8)$ gehalten. Und auf dem gleichen Boden steht schlieblich das Erlebnis der Eltern mit dem zwölfjährigen Sohne im Tempel $(2,4 \mathrm{lff})$, das bereits oben S. 9 besprochen ist.

Wir erkennen somit in dem Berichte des Lc einen judenchristlichen Versuch, die Geburt und Kindheit Jesus mit dem Glanz des Wunderbaren zu verklären, den seine Berufung zum Messias zu erfordern schien. Die Wunder aber beschränken sich ausschlieblich auf göttliche Offenbarungen, die von Engeln überbracht werden oder in den handelnden Personen durch die Kraft des göttlichen Geistes hervorbrechen. Die geschichtliche Überlieferung, auf welcher der Kern des Evangeliums stand, daß Jesus als ältestes Kind des Joseph und der Maria von Nazareth geboren war, ist noch treu festgehalten. Nur die Forderung, daß Jesus durch seinen Vater dem Hause David angehöre und in der Davidstadt Bethlehem geboren sei, war bereits für diese ganz durch die messianischen Vorstellungen beherrschte Dichtung unerläßliche Voraussetzung. Erst der Überarbeiter hat, indem er durch Einschaltung von $\mathrm{r}, 34 \mathrm{f}$. einen Kompromiß mit der durch Mt verbreiteten Sage schloß, einen fremdartigen, unvereinbaren $\mathrm{Zug}$ in die einheitlich gestaltete Dichtung bei Lc eingedrängt.

3. Dagegen ist der Bericht des Mt ganz beherrscht von der Voraussetzung, daß Jesus im jungfräulichen Leibe der Maria empfangen sei vom heiligen Geiste. Joseph erhält die Offenbarung, 'das in ihr Gezeugte ist aus dem heiligen Geiste'; und Joseph, der göttlichen Weisung folgend, 'erkannte sie nicht, bis sie einen Sohn geboren hatte'. Man kann die göttliche Zeugung als eine Rückübertragung der bei Lc hervortretenden Auffassung des Taufwunders betrachten. Aber es kommt etwas völlig Neues hinzu, die Empfängnis und Geburt der Jung frau. Und hiermit treten wir zweifellos auf den Boden heidnischer Vorstellungen. Schon die alten Väter der Kirche haben sich dieses Gefühls nicht erwehren können. Dem Judentume ist die Vorstellung ganz fremd, während sie bis über die augusteische Zeit dem griechisch-römischen Heidentume höchst lebendig geblieben ist. Den Nachweis habe ich früher gegeben und könnte ihn heute verstärken. Das Wort des Jesaias 7, I4 hat zur Gestaltung dieser Geburtssage um so weniger Veranlassung gegeben, als der Zusammenhang der Stelle gar nicht auf einen enwarteten Messias 
hinweist und nur von einem jungen Weibe, nicht einer Jungfrau, wie es in der LXX heißt, spricht. Die Anstrengungen, welche man gemacht hat, um das höchst unliebsame Eingreifen heidnischer Mythologie in den Stoff der Evangelien abzuwehren, sind vergeblich gewesen. Man hüte sich mit Belegen zu kommen, weiche das Gegenteil von dem beweisen, was sie sollen. An einer merkwürdigen Stelle (de Cherubim I3, I, p. I80 f. Cohn) sucht Philo, indem er die Ausdrucksweise der heiligen Schrift preßt, zu zeigen, daß es Gott gewesen, der Sarah, Lea, Rebekka und Sepphora befruchtet habe. Hier wird allerdings göttliche Zeugung, wenn auch nicht jungfräuliche Geburt, gelehrt. Aber man darf nicht übersehn, daß Philo diese Lehre als ein Mysterium, als eine weihevolle Offenbarung, mit anderen Worten als etwas ganz Neues bezeichnet; die neue Erkenntnis ist ihm erst in der hellenistischen Atmosphäre Alexandreias aufgegangen, an der Quelle aller der Ideen, womit er die Úberlieferungen seines Volkes zu vertiefen wußte.

Wie der Grundfaden des Gewebes, so sind auch alle Einschläge auf heidnischem Boden gewachsen. Die Erscheinung eines neuen Sterns am Himmel, der die Geburt des Heilands anzeigt, war im antiken Volksglauben vorgebildet. Von Astrologen wurde sogar gelehrt, daß bei der Geburt eines jeden Menschen ein neuer Stern emporsteige (s. Julianus Halik. im Rhein. Mus. 55, 328, Z. II). Noch an die Geburt des Alexander Severus ist die Sage geknüpft worden, daß durch die plötzliche Erscheinung eines Sternes erster Größe die künftige Weltherrschaft des Knäbleins verkündigt worden sei (Lampridius c. I3): das kann semitischer Herkunft sein. Auch $\mathrm{da} ß$ von den sternkundigen Magiern die Geburt des Heilands erkannt und verkündet wird, hat sein Vorbild in einer schon von Cicero (de divin. I 23, 47 vgl. 4I, 90) erwähnten Alexandersage. $\mathrm{Daß}$ sie aber selbst kommen, um den neuen Herrn zu verehren, scheint, wie unlängst in dieser Zeitschrift ( 3 , I ff.) A. Dieterich gezeigt hat, veranlabt $\mathrm{zu}$ sein durch die Huldigungsreise des Partherkönigs Tiridates zu Nero nach Rom, die im Jahre 66 n. Chr. das größte Aufsehn machte (s. Cassius Dio 63, 2 f.), vor allem in den Provinzen, welche, wie Kleinasien, den König mit seinem Troß zu sehen bekamen und standesgemäß zu unterhalten hatten; Plinius, der $n: h .30,16 \mathrm{f}$. dieses Ereignisses gedenkt, nennt den Tiridates geradezu magus und erwähnt, daß er Magier in seinem Gefolge gehabt habe (magos secum adduxerat), von denen der Kaiser die Geheimnisse der Magie zu erfahren hoffte. Die Regierung des Nero mag die Zeit sein, wo die Legende von der göttlichen Geburt Christi in dem christlichen Volk sich zu bilden 
begann "; und es ist selır möglich, daß die von Rom her verbreitete Kunde der Neronischen Cliristenverfolgung dazu beigetragen hat, das Bild des blutdürstigen Tyrannon in dic Kindheitsgeschichte Jesus' aufzunelmen. An sich war der Kindermord und seine Motivierung durch die Furcht vor cinem drohenden neuen Herrscher bereits in dem Sagenschatz vorhanden, wie die romanhafte Erzählung des Marathus von der Geburt des Augustus (Suetonius Aug. 94) lehrt.

Dic Flucht nach Ägypten oder, genauer gesagt, die Rückkehr aus Ägypten ist übel motiviert. Ein Engel des Herrn mahnt Joseph, wie vorher zur Errettung des Knäbleins vor Herodes, so zur Heimreise: 'denn sie sind gestorben, die dem Kinde nach dem Leben trachteten' (MIt 2, 20); allein 'da er hörte, daß Archelaos in Judäa an Stelle seines Vaters Herodes König sei, fürchtete er sich, dorthin zu gehn' und 'auf eine göttliche Weisung in Traume zog er sich in die Landschaft Galiläa zurück' (Mt 2, 22). Es wäre nicht recht zu begreifen, weshalb der Engel des Hcrrn nicht bis zur Absetzung des Archelaus (6n. Chr.) den Befehl zur Rückkehr vertagte, wenn nicht dadurch der Grund, Nazareth in Galiläa zum Wohnort zu wählen, weggefallen wäre. Aber um diesen Zweck zu erreichen, wird, schwerfällig genug, eine doppelte Offenbarung an Joseph insceniert. Warum ist es Ägypten, das als Zufluchtsort dient? Man könnte sagen, es habe im ersten Jahrhundert, wo längst Juden in großer Zahl sich zu Alexandreia gesammelt hatten, nahe gelegen an dies Nachbarland zu denken. Es kann auch durch unwillkürliche Ein-

I Eine apokryphe, syrisch in einer alten, aus dem VI Jahrh. stammenden Handschr. des Brit. Mus. erhaltene Schrift unter dem Namen des Eusebios, die W. Wright im Journal of sacred literature IX (Lond. I866) p. I17 ff. herausgegeben und ebend. X (1867) $151 \mathrm{ff}$. in englischer Übersetzung vorgelegt hat (vgl. auch den Aufsatz von Eb. Nestle, Zeitschr. f. wiss. Theologie 1893, 435 f.), berichtet, dab in der Zeit des Hadrian und des Papstes Xystus und zwar im J. $120 \mathrm{n}$. Chr. (das Jahr ist durch die römischen Consuln und das Jahr $43^{\circ}$ der Seleukidenära festgelegt) mit der h. Schrift beschäftigte Männer das Genauere über die Sendung der Magier in den persischen Archiven erforscht und in ihrer Sprache aufgezeichnet hätten. Conybeare hat mir die Freude gemacht, mich auf diese Nachricht aufmerksam zu machen. Der Zusammenhang des ganzen Traktats (leider ist gerade die den angezogenen Angaben rorausgehende Stelle, offenbar ihres haeretischen Inhalts wegen, getilgt worden) schließt den Gedanken aus, daß in das Jahr 120 die Einfügung der Magierepisode in das Evangelium verlegt werde. Es ist notwendig anzunehmen, dalj dem pseudonymen Verfasser ein ausführlicher in Persien spielender Bericht über die Erscheinung des Sterns und die Sendung der Magier vorlag, der jene (natürlich erdichtete) Datierung sei es im Eingang oder am Schluß hatte. Man denkt unwillkürlich an die Einlage des zuletzt von Bratke (Texte und Unters. N. F. IV, 3) herausgegebenen Religiongesprächs p. II ff., die zweifellos schon eine längere Vorgeschichte hatte, bevor sie vom Verfasser dieses Gesprächs verwertet wurde. 
wirkung mythischer Vorstellungen in die Legende eingefügt sein: auch vor dem Angriff des reckenhaften Typhon flüchten sich die olympischen Götter nach Ägypten.

Somit läßt sich für die ganze Geburts- und Kindheitsgeschichte des Mt bis in alle Einzelheiten heidnische Unterlage erweisen. Sie muß in heidenchristlichen Kreisen, wahrscheinlich Kleinasiens entstanden sein, und wurde dann vom Erzähler, entsprechend der das ganze Ev. Mt beherrschenden Neigung (s. Resch, Kindheitsevang. S. I9 ff.) durch Heranziehung von Prophetenworten gewissermaßen legitimiert.

Damit war die göttliche Geburt Christi für alle Zeit besiegelt, und die judenchristliche Darstellung des Lc, welche den Messias nur als einfachen. Menschensohn kannte, mußte durch die Erweiterung der englischen Botschaft auf die Höhe der Zeit gehoben und mit den Forderungen des Glaubens in Einklang gesetzt werden.

Die göttliche Geburt Christi war damit Evangelium geworden. Der theosophischen Spekulation erwuchs die Aufgabe, dies Dogma mit der Tatsache der Menschlichkeit Christi auszugleichen. Es kostete einen Kampf von Jahrhunderten, bis die Kirche eine einheitliche Glaubenslehre durchgesetzt hatte. Freilich dieser Kampf würde entbrannt sein, auch wenn das Evangelium von der jungfräulichen Geburt nicht geschrieben vorgelegen hätte. Schon bevor dies geschrieben und durchgedrungen war, hatte sich die doketische Lehre, daß der Sohn Gottes vom Himmel herabgesandt nur ein Scheinleben als Mensch geführt habe, und die Johanneische Vorstellung von der Präexistenz des göttlichen Logos entwickelt. 